JOURNAL OF

SYMPLECTIC GEOMETRY

Volume 3, Number 4, 565-587, 2005

\title{
STEIN SURFACES AS OPEN SUBSETS OF $\mathbb{C}^{2}$
}

\author{
Robert E. GOMpF
}

An open subset $\boldsymbol{U}$ of a complex surface can be topologically perturbed to yield an open subset whose inherited complex structure is Stein, if and only if $\boldsymbol{U}$ is homeomorphic to the interior of a handlebody whose handles all have index $\leq \mathbf{2}$.

\section{Introduction}

Stein surfaces have been intensively studied in some form by complex analysts since the early 20th century. The most basic question, how plentiful Stein surfaces are, still remains wide open in various ways. For example, any open subset $U$ of a complex surface $X$ inherits a complex structure, so one may ask how commonly open subsets of $X$ satisfy the Stein condition. This is already a deep classical problem when $X=\mathbb{C}^{2}$. In the present paper, we show that Stein open subsets of a complex surface $X$ are ubiquitous in the following sense: if $U$ satisfies the most basic topological condition necessary for the existence of a Stein structure, then after a suitable adjustment it becomes Stein as an open subset of $X$. The adjustment is quite mild from a point-set topological viewpoint - in particular, the homeomorphism type of $U$ is preserved, as well as the essential data of the topological embedding in $X$. However, the differential topology is radically altered. The diffeomorphism type of $U$ is typically changed, and frequently there are uncountably many possibilities for the resulting diffeomorphism type. The purpose of this paper is to state and prove the simplest form of the above existence result (Theorem 2.4), while developing the necessary background material. We also describe stronger results which will be presented in a forthcoming paper.

\section{Stein surfaces and where to find them}

There are many equivalent ways of defining Stein surfaces. Perhaps the most efficient is that a Stein surface is a complex surface (so the real 
dimension is 4) that admits a biholomorphic embedding as a closed subset of $\mathbb{C}^{N}$ for some $N$. (A more relevant characterization will be given in Section 4.) By the maximum modulus principle, Stein surfaces are never compact, but they might be considered the most natural generalization of compact Kähler surfaces. Global analysis such as Seiberg-Witten theory extends from the compact setting to the Stein setting [16], because the Stein condition imposes "pseudoconvexity" at infinity, which has the effect of allowing suitable boundary conditions for the analysis. There is an analogous notion of convexity in symplectic geometry, leading to the concept of a Weinstein symplectic manifold $[\mathbf{6}, \mathbf{2 2}]$, but for our present purposes, the complex analytic setting is more natural. (We return to the Weinstein case in the last paragraph of the paper.) It is important to note that, unlike affine algebraic surfaces, Stein surfaces need not have finite topology. Equivalently, a Stein surface need not be diffeomorphic to the interior of a compact 4-manifold with boundary. In fact, infinite topology is central to the construction in this paper, so the resulting smooth manifolds are typically "exotic." To apply Seiberg-Witten theory to such manifolds, for example, one must observe that they can be written as infinite nested unions of Stein surfaces with finite topology. (This at least gives information about compactly supported objects, for example, the minimum genus of a representative of a fixed homology class.)

The most basic question we can ask about Stein surfaces is how plentiful they are. We can make this question less vague in two different ways - considering Stein surfaces as abstract manifolds or as open subsets of complex surfaces.

\section{Questions 2.1.}

(a) Which open, oriented 4-manifolds admit Stein structures?

(b) In a fixed complex surface $X$, how common are open subsets $U$ that are Stein surfaces in the complex structure inherited from $X$ ?

The second question is already interesting for the simplest complex surface $X=\mathbb{C}^{2}$. Such open subsets are classically called "domains of holomorphy."

To address these questions, it is convenient to use the language of handlebody theory. Recall that a $k$-handle attached to the boundary of an $n$-manifold is a "thickened up" $k$-cell, that is, a copy of $D^{k} \times D^{n-k}$ smoothly attached along $\partial D^{k} \times D^{n-k}$, with the corners smoothed. The central $k$-cell $D^{k} \times\{0\}$ is called the core of the handle. A handle decomposition is a decomposition of a manifold into handles, essentially a thickened up $C W$-complex, cf. Figure 1. In the context of open manifolds, we build the handlebody first, then remove its boundary. Handlebody theory is equivalent to Morse theory: a proper Morse function to $[0, \infty)$, such as height in Figure 1, determines a 

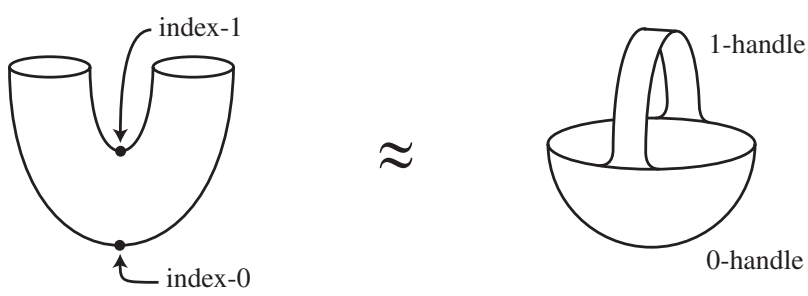

Figure 1. Handle decomposition of $S^{1} \times \mathbb{R}$.

handle decomposition on its domain, with each index- $k$ critical point determining a $k$-handle [18]. This shows that all smooth manifolds admit handle decompositions.

Question 2.1(a) was answered by Eliashberg [5], at least in principle. His theorem is cleanest for higher dimensional Stein manifolds (complex $n$-manifolds embedding as closed subsets of $\left.\mathbb{C}^{N}\right)$. It was classically known that every Stein manifold $X$ admits a proper Morse function to $[0, \infty)$ with critical points whose indices never exceed the complex dimension, or equivalently, a handle decomposition whose indices never exceed the middle (real) dimension. In addition, the complex structure on $X$ determines an almost-complex structure (complex vector bundle structure on $T X$ ). Surprisingly, these conditions are also sufficient for the existence of a Stein structure in high dimensions.

Eliashberg's Theorem 2.2 [5]. For $n \geq 3$, let $X$ be a smooth $2 n$-manifold with an almost-complex structure $J$. If $X$ is the interior of a (possibly infinite) handlebody whose indices are all $\leq n$, then $X$ admits a Stein structure homotopic to $J$.

Since the existence of an almost-complex structure is a homotopy-theoretic question, Eliashberg's theorem characterizes manifolds admitting Stein structures in purely topological terms. The method also applies when $n=2$ (and the statement is well known when $n=1$ ). In the $n=2$ case, almostcomplex structures always exist (since we are dealing with oriented manifolds homotopy equivalent to 2-complexes). However, a framing obstruction arises, which always vanishes for $n \geq 3$, but is nontrivial for 2-handles on 4-manifolds. Thus, Eliashberg characterizes oriented 4-manifolds admitting Stein structures as being interiors of handlebodies with all indices $\leq 2$ and an additional condition on the normal twisting of the 2-handle attaching maps (see e.g., [12] for a careful statement). While this is a purely topological characterization (and can be expressed entirely in terms of Kirby diagrams), it is somewhat difficult to apply in practice (e.g., $[\mathbf{1 2}, \mathbf{1 4}])$.

It is natural to ask whether the $n=2$ case of Theorem 2.2 might be true as stated, even though it doesn't follow from Eliashberg's method. In fact, it 
already fails for the simplest example with a 2-handle: $S^{2} \times \mathbb{R}^{2}$ clearly satisfies the hypotheses of the theorem, being a 0 -handle with a 2-handle attached in the simplest way. It is even complex $\left(\mathbb{C P}^{1} \times \mathbb{C}\right)$. However, Seiberg-Witten theory $[\mathbf{1 6}, \mathbf{1 7}]$ implies that any smoothly embedded, homologically essential sphere in a Stein surface must have homological self-intersection number $\leq-2$, so $S^{2} \times\{0\} \subset S^{2} \times \mathbb{R}^{2}$ rules out the existence of a Stein structure. On the other hand, we might take inspiration from Freedman's revolutionary work $[\mathbf{1 0}, \mathbf{1 1}]$ : while the main theorems of high-dimensional differential topology fail miserably in dimension 4 , they tend to work if we ignore smooth structures and analyze the underlying topological manifolds. By combining Freedman's machinery with Eliashberg's, one obtains Theorem 2.2 up to homeomorphism.

Theorem 2.3 [12]. An oriented, topological 4-manifold $X$ is homeomorphic to a Stein surface if and only if it is the interior of a (possibly infinite) topological handlebody with all indices $\leq 2$. In fact, every homotopy class of almost-complex structures on $X$ is then realized by such (orientationpreserving) homeomorphisms.

In dimension 4, topological handlebodies are uniquely smoothable (since we are gluing along 3 -manifolds). See $[\mathbf{1 2}]$ for why almost-complex structures can be pulled back through homeomorphisms, up to homotopy. This theorem implies, for example, that there are Stein surfaces homeomorphic to $S^{2} \times \mathbb{R}^{2}$ (but of course not diffeomorphic to it), and in fact their Chern classes (hence, canonical classes) realize all even multiples of the generator of $H^{2}\left(S^{2} \times \mathbb{R}^{2} ; \mathbb{Z}\right)$.

To address Question 2.1(b), we first observe that an open set $U \subset X$ has no chance of being Stein unless it has a handle decomposition with all indices $\leq 2$. Thus it suffices to consider open sets obtained by suitably thickening embedded 2-dimensional $C W$-complexes. For a topologically embedded 2-complex $K \subset X$, a neighborhood $U$ of $K$ will be called a thickening of $K$ if there is a homeomorphism $h: U \rightarrow$ int $H$ onto the interior of a handlebody, sending cells to cores of handles. That is, there is a bijection between $k$-cells of $K$ and $k$-handles of $H$ for each $k$, and $h$ sends each cell to the core $D$ of the corresponding handle together with a collar of $\partial D$ that is radial with respect to the normal disks $\{p\} \times D^{4-\ell}$ of the lower index handles (Figure 2). A complex $K \subset X$ will be called tame if a thickening exists. This rules out wild embeddings analogous to Alexander's horned sphere. Any two thickenings of a fixed $K \subset X$ will be homeomorphic (rel $K$ and preserving handles), although not necessarily diffeomorphic. We can ask whether they are topologically ambiently isotopic (so they correspond under a homeomorphism $X \rightarrow X$ that is isotopic to $\mathrm{id}_{X}$, i.e., homotopic to $\mathrm{id}_{X}$ through homeomorphisms). We may first need to topologically radially shrink the thickenings 


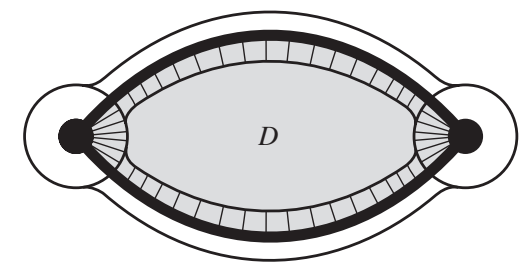

Figure 2. Core $D$ and its collar, comprising a 2-cell (shaded).

slightly to avoid technical difficulties at the boundary. (For example, the annuli $a<r<b$ in $\mathbb{R}^{2}$ are ambiently isotopic to each other for all $a, b>0$, but the case $a=0$ poses difficulties.) Then it seems plausible that the thickenings should be ambiently isotopic, but the question is apparently still open. Our main theorem now gives an answer to Question 2.1(b), by asserting that Stein open subsets $U \subset X$ are as common as possible, up to shrinking at the boundary and topological ambient isotopy. That is, the existence of any thickening implies (up to isotopy) the existence of a Stein thickening isotopic to it after shrinking.

Main Theorem 2.4. Let $K \subset X$ be a tame, topologically embedded $C W$ 2-complex in a complex surface, and choose $\varepsilon>0$. Then after an $\varepsilon$-small topological ambient isotopy of $K$, an $\varepsilon$-neighborhood of $K$ contains a thickening $U$ of $K$ that is Stein in the complex structure that $U$ inherits as an open subset of $X$.

By construction, the Stein thickening $U$ becomes topologically ambiently isotopic to the originally chosen thickening $V$, once we shrink $U$ and $V$ at their boundaries. A fancier construction $[\mathbf{1 3}]$ renders the shrinking unnecessary, provided that $\partial V$ is suitably tame. If $K$ is infinite, we may take $\varepsilon$ to be a positive function on a neighborhood of $K$.

\section{Examples 2.5.}

(a) We consider $S^{2}$, the simplest complex of dimension 2. A smooth 2-knot is a smooth embedding $S^{2} \hookrightarrow \mathbb{R}^{4}$. The theory of smooth 2 -knots $K$ is even richer than classical knot theory. For example, all classical knot groups (as well as other groups) arise as smooth 2-knot groups $\pi_{1}\left(\mathbb{R}^{4}-K\right)$, and $\pi_{2}$ plays a role as well. For any 2 -knot $K \subset \mathbb{C}^{2}=\mathbb{R}^{4}$, the theorem supplies a Stein surface $U \subset \mathbb{C}^{2}$ that remembers the original knot type. For example, $K$ and the closure of $U$ have complements with the same homotopy groups (although one must look carefully at the proof to see this, due to possible complications along the boundary of $U$ ). The Stein surface $U$ is homeomorphic to $S^{2} \times \mathbb{R}^{2}$ (since $K$ clearly has vanishing homological self-intersection number, so its normal bundle is trivial). Thus we obtain a plethora of counterexamples 
to the conjecture of $[\mathbf{8}]$ that no Stein open subset of $\mathbb{C}^{2}$ is homotopy equivalent to $S^{2}$. However, $U$ is clearly not diffeomorphic to $S^{2} \times \mathbb{R}^{2}$, since $H_{2}(U)$ cannot be generated by a smoothly embedded sphere. (This shows the necessity of the topological isotopy $-U$ cannot contain the original smooth sphere $K$ or anything smoothly isotopic to it, although by construction, it contains a topological sphere topologically isotopic to $K)$. One can arrange for the minimal genus of a smooth surface generating $\mathrm{H}_{2}(U)$ to be any preassigned positive integer. Thus, each 2-knot $K$ corresponds to infinitely many diffeomorphism types of Stein surfaces $U$, distinguished by the minimal genus of the generator. On the other hand, one can identify a single diffeomorphism type that can be realized by Stein thickenings (after topological isotopy) of all smooth 2-knot types, and such universal diffeomorphism types can be found with arbitrary nonzero minimal genus. It is not known if topological 2-knots $S^{2} \hookrightarrow \mathbb{C}^{2}$ exist that have topological $S^{2} \times \mathbb{R}^{2}$ thickenings but cannot be smoothed by a topological isotopy. If so, one would expect that no universal diffeomorphism types exist for their Stein thickenings, but any finite collection of topological 2-knots must (after isotopy) have a common diffeomorphism type of Stein thickenings.

(b) To get more complicated embeddings $S^{2} \hookrightarrow \mathbb{C}^{2}$, start with a classical knot $\kappa$ (smooth embedding $S^{1} \hookrightarrow S^{3}$ ), and let $H_{\kappa}$ denote the handlebody obtained by attaching a 2-handle to a 0-handle $D^{4}$ along $\kappa \subset \partial D^{4}$, with the normal directions to $\kappa$ matched up so that $H_{\kappa}$ has trivial intersection pairing. The knot $\kappa$ is called (topologically or smoothly) slice if $H_{\kappa}$ embeds (topologically or smoothly) in $\mathbb{R}^{4}$ (or equivalently in $S^{4}$ ). For example, if the Alexander polynomial $\Delta_{\kappa}(t)$ is 1 , then $\kappa$ is topologically slice with $S^{4}-H_{\kappa}$ homotopy equivalent to $S^{1}$ [11]. An embedding of $H_{\kappa}$ determines an embedding $K$ of $S^{2}$ with a unique locally knotted point $p$, at which we see the cone on $\kappa$ in $D^{4}$. If we identify $K$ with the $C W$-complex $\{p\} \cup 2$-cell, then int $H_{\kappa}$ is a thickening of $K$. For a fixed topologically slice $\kappa$, we obtain an entire knot theory for such singular 2-spheres $K$. For example, if $\Delta_{\kappa}(t)=1$, we obtain topological knot complements in $\mathbb{R}^{4}$ realizing all the homotopy types of smooth 2-knot complements, by ambiently connected summing smooth 2 -knots with the homotopy- $S^{1}$ case mentioned above. Many knots with $\Delta_{\kappa}(t)=1$ are known not to be smoothly slice, so the corresponding singular topological 2-knots $K$ can never be isotoped to singular smooth 2-knots whose singularities are smooth cones. (That is, the embedded tame 2-complexes $K$ are unsmoothable.) However, the theorem still applies, and the discussion of (a) goes through without significant change, except that the resulting Stein surfaces in $\mathbb{C}^{2}$ will all be homeomorphic to int $H_{\kappa}$ rather than to $D^{2} \times \mathbb{R}^{2}$, while 
they are still homotopy equivalent to $S^{2}$. (The minimal genus can still be taken to be arbitrarily large, although we lose precise control over it, and we still obtain universal diffeomorphism types in the smooth setting for a fixed, smoothly slice $\kappa$.)

The multitude of smooth structures we encounter here leads us to consider the quintessential phenomenon of open 4-manifold smoothing theory: exotic $\mathbb{R}^{4}$ 's. These are smooth manifolds homeomorphic to $\mathbb{R}^{4}$ but not diffeomorphic to it. In contrast to dimensions $n \neq 4$, where exotic $\mathbb{R}^{n}$ 's cannot exist, exotic $\mathbb{R}^{4}$ 's realize uncountably many diffeomorphism types. (See Section 9.4 of $[\mathbf{1 4}]$ for a survey.) It was shown in $[\mathbf{1 2}]$ that uncountably many exotic $\mathbb{R}^{4}$ 's admit Stein structures. These smooth manifolds embed smoothly in the standard $\mathbb{R}^{4}$. Subsequently, Taylor $[\mathbf{2 1}]$ showed that uncountably many exotic $\mathbb{R}^{4}$ 's (that do not embed in $\mathbb{R}^{4}$ ) require 3 -handles in their handle decompositions, so they cannot support Stein structures. The techniques of the present paper can be used to prove the following theorem. (Details will appear in $[\mathbf{1 3}]$.)

Theorem 2.6. There is a family of open subsets of $\mathbb{C}^{2}$ (with compact closure) that are Stein and homeomorphic to $\mathbb{R}^{4}$, but realize uncountably many diffeomorphism types (with the cardinality of the continuum in ZFC set theory).

The usual way of finding an uncountable family of exotic $\mathbb{R}^{4}$ 's is to identify one exotic $\mathbb{R}^{4}$ topologically with $\mathbb{R}^{4}$, then consider open balls of all sufficiently large radii. These topological balls inherit exotic smooth structures that can frequently be distinguished. The family in the above theorem arises in such a manner, with the Stein open subsets corresponding to radii lying in a Cantor set. Once we have such a family, we can connect it by a 1-handle to any $U$ as in Theorem 2.4 and obtain a version of:

Theorem 2.7. For $K \subset \mathbb{C}^{2}$ nonempty, the neighborhood $U$ given by Theorem 2.4 lies in a family of Stein thickenings of $K$, nested (with compact closure in each other if $K$ is compact) with the order type of a Cantor set, and realizing uncountably many diffeomorphism types. Every nonempty Stein open subset $U \subset \mathbb{C}^{2}$ contains an uncountable nested family of Stein open subsets homeomorphic to $U$, but with no two diffeomorphic.

The structure of such families will be considered in more detail in Section 7 and in [13]. Similar (and sometimes stronger) results can be obtained for other cases of $K \subset X$. One would expect to essentially always obtain such uncountable collections of diffeomorphism types, but distinguishing these in complete generality seems beyond the range of present technology.

Example 2.8. For an embedded sphere $K \subset \mathbb{C}^{2}$ as in Example 2.5(a) or (b), we obtain an uncountable family of nested Stein surfaces corresponding to 
the given knot type, realizing uncountably many diffeomorphism types. This phenomenon is independent of the countably infinite families of the previous examples: we can find a family as in Theorem 2.7 for which the minimal genus of a generator is any fixed $g \geq 1$ in (a) and fixed but arbitrarily large in (b), or arrange the minimal genus to increase without bound as the Stein surfaces get smaller. We can assume the intersection of the Stein surfaces is a sphere topologically ambiently isotopic to $K$ and that this is smooth except at a single point (aside from the smooth cone point $p$ in (b)).

Most of the remainder of this paper is devoted to a proof of Main Theorem 2.4, together with relevant background material. In the final Section 7, we will comment on the proofs of some of the remaining claims of this section and present some additional observations. More systematic and general statements and proofs will appear in $[\mathbf{1 3}]$.

\section{Totally real surfaces}

Our first tool for proving Main Theorem 2.4 involves embedded surfaces. Suppose $F$ is a compact, connected, oriented surface (so $\operatorname{dim}_{\mathbb{R}} F=2$ ) smoothly embedded (or immersed) in a complex surface $X$. It is natural to ask how the complex structure of $X$ relates to $F$. We observe that there may be points $x \in F$ at which the (unoriented) tangent space $T_{x} F$ is a complex line; we call these complex points. Generically, $F$ has only finitely many complex points. This suggests counting complex points with suitable signs to obtain an index. We can define the sign of a complex point to be $(+)$ if and only if the complex orientation agrees with that of $F$. However, there is also a second, more subtle, notion of sign, leading to the distinction between elliptic and hyperbolic points (which should also be familiar to contact geometers) and resulting in two independent integer invariants (tracing back, e.g., to $[\mathbf{3}])$. While the details are not crucial for this paper, we state them for completeness: the Grassmann bundle $\operatorname{Gr}(T X)$ of oriented real 2planes on $X$ has a pair $\mathbb{P}_{ \pm}(T X)$ of codimension- 2 subbundles, consisting of complex lines, positively and negatively oriented. The bundle $T F$ can be interpreted as a section of $\operatorname{Gr}(T X)$ over $F$, so it has intersection numbers with $\mathbb{P}_{ \pm}(T X)$ yielding the required pair of integers.

These invariants, repackaged by linear combination, can be recognized as familiar characteristic classes: the Chern number $\left\langle c_{1}(X), F\right\rangle$ and the sum $e(\nu F)+\chi(F)$ of normal and tangent Euler numbers. If $F$ is totally real, i.e., if $F$ has no complex points, then both invariants must vanish by definition. By a harder result of Eliashberg and Harlamov, these are the only obstructions.

Theorem 3.1 [7]. A surface $F \subset X$ as above is smoothly isotopic to a totally real surface if and only if $\left\langle c_{1}(X), F\right\rangle=0=e(\nu F)+\chi(F)$. 
In fact, when both invariants vanish, Eliashberg and Harlamov connect canceling pairs of complex points by arcs in $F$ and then eliminate the complex points by a $C^{0}$-small twist of $F$ fixing each arc but rotating its normal directions. (See also $[\mathbf{1 9}]$ for more details and $[\mathbf{8}]$ for a more general version.) The theorem is intended to apply rel boundary when $\partial F \neq \emptyset$. In that case, one needs suitable framings on $\partial F$ to interpret the characteristic classes. Generically, $F$ has no complex points on $\partial F$, so the tangent and outward normal vector fields $t$ and $n$ along $\partial F$ are a complex framing of $T X \mid \partial F$ for defining $\left\langle c_{1}(T X), F\right\rangle$, and multiplying by $i$ sends $t$ to a normal vector field to $F$ along $\partial F$ defining the relative Euler class $e(\nu F)$. (Of course, the topological Euler characteristic $\chi(F)$ is also the tangent Euler number relative to $t$ in this case.)

\section{Smooth Stein thickenings}

Our method for constructing Stein surfaces is taken from Eliashberg's paper [5] proving Theorem 2.2. We will illustrate the method by attempting to prove a smooth version of Main Theorem 2.4- so the open cells of $K$ are smoothly embedded, the isotopy is smooth, and the thickenings come from smoothly embedded handlebodies. We are doomed to fail, even for the simple case $K=S^{2} \subset \mathbb{C}^{2}$, but the method works for some pairs $K \subset X$. It is instructive to see how the method fails in general, so we can ultimately fix the proof by passing to the topological setting and invoking Freedman theory.

The Morse functions $\varphi$ discussed prior to Eliashberg's Theorem 2.2 (with all indices $\leq \operatorname{dim}_{\mathbb{C}} X$ ) are actually plurisubharmonic functions. These are essentially characterized as having level sets $\varphi^{-1}(c)$ that are all pseudoconvex. In complex dimension 2 , this is the same as saying that the complex line field determined on each $\varphi^{-1}(c)$ (by the complex structure) is a contact structure that is positive in the boundary orientation on $\varphi^{-1}[0, c]$. (See, e.g., [12] or Chapter 11 of [14] for further discussion.) By a theorem of Grauert [15], a complex manifold with a proper plurisubharmonic function to $[0, \infty)$ must be Stein, so Stein manifolds are characterized by the existence of these functions. Eliashberg builds his Stein manifolds as complex manifolds with plurisubharmonic Morse functions, extending the complex structure and function one handle at a time. In the case of Stein open subsets ([5], Theorem 1.3.6), the complex structure is inherited from a carefully chosen embedding of the handle into the ambient manifold.

Eliashberg's method is simplest for 0- and 1-handles. In our case, we start with a smooth 2-complex $K \subset X$ in a complex surface. First thicken each 0 -cell to a standard closed $\varepsilon$-ball in some local holomorphic coordinates. Such a ball $B$ automatically has a pseudoconvex boundary that is a level set of the plurisubharmonic function $\varphi(z)=\|z\|^{2}$ in the local chart 
near $B$. We can assume these balls are the 0-handles of our given smooth thickening of $K$, after a smooth isotopy of the thickening. Now each 1-cell of $K$ determines an embedding $\gamma: I=[0,1] \rightarrow X$ whose image intersects the 0 -handles precisely at $\gamma(\partial I)$. After a $C^{1}$-small smooth isotopy fixing $\gamma$ and $d \gamma$ on $\partial I$, we can assume $\gamma$ is real-analytic. Then complexifying $\gamma$ gives a holomorphic embedding from a neighborhood of $I$ in $\mathbb{C}$. Using a realanalytic vector field $v$ on $\gamma(I)$ normal to this complex curve, we can extend to a neighborhood of $I \times\{0\}$ in $\mathbb{R}^{2}$, then complexify to a neighborhood in $\mathbb{C}^{2}$. This neighborhood of $I \times\{0\}$ in $\mathbb{C}^{2}$ is Eliashberg's model complex 1-handle, on which he explicitly constructs a plurisubharmonic extension of $\varphi$. (Here we also need the 0 -handle boundaries to intersect $I \times\{0\}$ orthogonally in the local model. This is easily achieved by suitable initial choice of $d \gamma$ and $v$ at $\partial I$.) After trimming each 1-handle back to a level set of $\varphi$, we have a Stein thickening $U_{1}$ of the 1-skeleton of $K$, and we can assume this agrees with the 0- and 1-handles of our original thickening (after an isotopy of the latter). This thickening $U_{1}$ has a natural boundary $\partial U_{1}$, which is a level set of the plurisubharmonic function $\varphi$ defined near $U_{1}$. (If $K$ has infinitely many 0 -cells, properness requires $\varphi$ to take arbitrarily large values on some 0 -cells, so in practice we should add some 0-handles after we have begun adding 1-handles if we wish $\varphi$ to be proper. This causes no difficulties. We also add a thin collar at each stage to arrange $\varphi$ to be well-defined on the infinite union. Note that $K$ is at least locally finite since it embeds in a manifold.)

Handles of larger index involve additional complications; for us, it suffices to consider a 2-handle. Each 2-cell of $K$ determines a smooth embedding $f: D^{2} \rightarrow X$ with $f^{-1}\left(\partial U_{1}\right)=\partial D^{2}$, which we would like to identify with the core of a model complex 2-handle, $D^{2} \times\{0\} \subset D^{2} \times D^{2} \subset \mathbb{R}^{2} \times i \mathbb{R}^{2} \cong \mathbb{C}^{2}$. This is only possible if $f\left(D^{2}\right)$ suitably resembles $D^{2} \times\{0\}$ in the model-for example, it should be totally real. (The model is chosen this way since the natural descending disks of a plurisubharmonic function are totally real. Further motivation is that every totally real submanifold $R$ has Stein tubular neighborhoods, since the squared distance to $R$ in any Hermitian metric is plurisubharmonic near $R$. Note that in the previous paragraph, the 0 - and 1-cells were automatically totally real.) First we adjust $\partial f\left(D^{2}\right)$. After a $C^{1}$-small perturbation, we can assume $\partial U_{1}$ is real-analytic near $\partial f\left(D^{2}\right)$. A $C^{0}$-small isotopy of $\partial f\left(D^{2}\right)$ in $\partial U_{1}$ makes it Legendrian, i.e., tangent to the contact planes of $\partial U_{1}$, and [5, Lemma 2.5.1] a further $C^{1}$-small perturbation makes $f \mid \partial D^{2}$ also real-analytic. The Legendrian condition implies $f\left(D^{2}\right)$ has no complex points on its boundary, so the invariants of Theorem 3.1 are defined. If these obstructions vanish, then we can isotope $f$ rel $\partial D^{2}$ to a totally real embedding. A $C^{1}$-small isotopy fixing $f$ and (if realanalytic) $d f$ on $\partial D^{2}$ now makes $f$ real-analytic as well. (This follows from a general theorem, but for $D^{2}$ it can also be proved using Fourier series.) 
Now $f$ complexifies to a holomorphic embedding of a model 2-handle, and Eliashberg's construction gives the required plurisubharmonic extension of $\varphi$ as before. (The contact condition and suitable initial choice of outward normal to $f\left(D^{2}\right)$ along $\partial f\left(D^{2}\right)$ guarantee that $\partial U_{1}$ is orthogonal to $D^{2} \times\{0\}$ in the local model as required.)

In conclusion, we can smoothly isotope $K$ and give it a Stein thickening, provided that the two integer obstructions of Theorem 3.1 vanish for each 2-cell of $K$. When can we arrange this? Note that the obstructions are not well-defined until we fix a choice of the Legendrian curve $C=\partial f\left(D^{2}\right)$, since complex points can initially slide across the boundary. If we make one choice of $C$ and extend its tangent vector field $t$ to a section $v$ of the contact plane field near $C$ in $\partial U_{1}$, we can change to a different choice $C^{\prime}$ by adding small spirals to $C$ parallel to the contact planes (winding relative to $v$ ), changing the frame $(t, n)$ used to define the relative characteristic classes. We can force $\left\langle c_{1}(X), F\right\rangle$ to vanish by this trick. (In fact, $\left\langle c_{1}(X), f\left(D^{2}\right)\right\rangle$ can be interpreted as the rotation number of the Legendrian curve $C$ relative to some $v$; it is well-known that this can be changed arbitrarily by adding zigzags to the diagram.) The other invariant $e(\nu F)+\chi(F)=e\left(\nu f\left(D^{2}\right)\right)+1$ is more problematic. (Its vanishing translates to the condition that the normal framing must equal $t b(C)-1$, the extra requirement arising in the $n=2$ case of Eliashberg's Theorem 2.2; cf. $[\mathbf{1 2}, \mathbf{1 4}]$.) This invariant vanishes mod 2 when the Chern obstruction does, since both count complex points with suitable signs. We can increase it by 2 without changing $\left\langle c_{1}(X), f\left(D^{2}\right)\right\rangle$, by adding an up/down pair of left-handed spirals (zig-zags), but cannot always decrease it. This is the fundamental flaw of the method, which prevents us from solving cases like $S^{2} \subset \mathbb{C}^{2}$. It is possible to solve some explicit cases, by directly computing the invariants and arranging them to vanish, but one cannot expect completely general results from this approach.

\section{Casson handles}

We now introduce the necessary elements of topological 4-manifold theory, with historical motivation. By the 1960s, differential topology in high dimensions $(\geq 5)$ had become a mature field, thanks to powerful theorems for reducing topological questions to algebraic ones - notably, the Surgery and $s$-Cobordism Theorems. The proofs of these theorems failed in dimension 4 , and little was known about 4-manifolds before 1980. The failure of the proofs can be traced to a key step called the Whitney trick, which allows us to separate intersecting submanifolds of complementary dimension. Under suitable hypotheses, we can group the extra intersection points in pairs with opposite sign, then connect each pair by an arc in each submanifold, to obtain a circle $C$ as in Figure 3(a). 
(a)

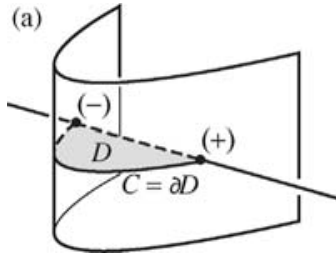

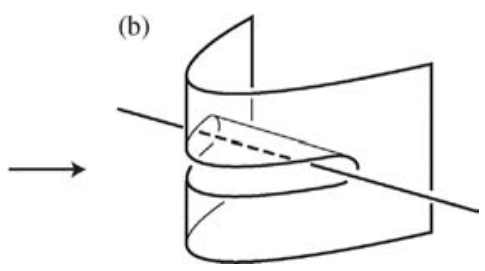

Figure 3. The Whitney trick.

If we can find an embedded 2-disk $D$ with $\partial D=C$ and int $D$ disjoint from the submanifolds, then we can eliminate the pair of intersections by pushing one submanifold across $D$ as in Figure 3(b). (One must also pay attention to the directions normal to $D$, but that obstruction always vanishes in high dimensions.) In high dimensions, one can find the disk $D$ when needed: The hypotheses guarantee that $C$ is nullhomotopic in the complement of the submanifolds, and the resulting map of a disk can be assumed to be a smooth embedding by transversality $(2+2<5)$. In dimension 4 , however, the best one can obtain by transversality is a smoothly immersed disk with transverse double-point intersections. Thus the fundamental question of 4-manifold theory is when immersed disks can be replaced by embedded disks, or equivalently, embedded 2-handles.

A major assault on this problem was launched by Casson [2] in the 1970s. As he observed, a kinky handle $\kappa$, or closed tubular neighborhood of a generically immersed 2-disk in a 4-manifold, is most obviously distinguished from a 2-handle by its fundamental group: Each kink (selfintersection) contributes a generator to this free group. One can recover a contractible space by attaching a 2-cell for each kink. In fact, Casson located a framed link $L$ in $\partial \kappa$, disjoint from the attaching region $\partial_{-} \kappa$, such that attaching 2-handles along $L$ transformed $\left(\kappa, \partial_{-} \kappa\right)$ into a 2-handle $\left(D^{2} \times D^{2}, S^{1} \times D^{2}\right)$. We can now rephrase the fundamental question: given an embedding $\left(\kappa, \partial_{-} \kappa\right) \rightarrow(X, \partial X)$ (where we can take the 4-manifold $X$ to be the complement of a neighborhood of the surfaces we wish to separate by the Whitney trick), when can we find embedded 2-handles in $X$ - int $\kappa$ attached along $L$ ? Such 2-handles would transform $\kappa$ into the desired 2-handle. Unfortunately, the new embedding problem seems even harder than the original. While this may have stopped lesser mathematicians, Casson proceeded to show that, after suitable modification of the original immersion, one can at least find disjoint kinky handles attached to $L$. The resulting union with $\kappa$ is now called a 2-stage Casson tower $T_{2}$. (One must use care with the meaning of attaching a kinky handle with a given framing, but this need not concern us here.) There is a new framed link on the boundary of the new kinky handles, on which attaching 2-handles would collapse the entire tower down to a 2-handle; Casson at 
least obtained disjoint kinky handles there for a 3-stage Casson tower $T_{3}$ in $(X, \partial X)$. By iterating the procedure, he obtained $n$-stage towers for all $n$, $\kappa=T_{1} \subset T_{2} \subset T_{3} \subset \cdots \subset X$. The union of these towers, with the boundary removed except for int $\partial_{-} \kappa$, is now called a Casson handle $\left(\mathrm{CH}, \partial_{-} \mathrm{CH}\right)$.

Why should we care about such a complicated infinite construct? First note that while each $T_{n}$ has a free fundamental group (generated by the top-stage kinks), a Casson handle is simply connected: any loop $\gamma$ in $\mathrm{CH}$ lies in some $T_{n}$ by compactness, but inclusion $T_{n} \hookrightarrow T_{n+1}$ is trivial in $\pi_{1}$. (The generators at each stage are killed by disks at the next stage.) Casson's more careful analysis showed that the smooth manifold $\left(\mathrm{CH}, \partial_{-} \mathrm{CH}\right)$ has the same proper homotopy type as an open 2-handle $\left(D^{2} \times \mathbb{R}^{2}, S^{1} \times \mathbb{R}^{2}\right)$. At this point, Freedman picked up the project. After most of a decade studying the internal structure of Casson handles, and the application of some difficult point-set topology, Freedman proved his amazing theorem.

Freedman's Theorem $\mathbf{5 . 1}[\mathbf{1 0}]$. Every Casson handle is homeomorphic to an open 2-handle.

Suddenly the Whitney trick and high-dimensional topology worked for topological 4-manifolds (first for trivial $\pi_{1}$, then later for many other fundamental groups [11]). Immediate consequences included the 4-dimensional Poincaré Conjecture, a complete classification of closed, simply connected topological 4-manifolds, and a Fields Medal for Freedman. (Some credit is also due for Quinn [20], for strengthening the conclusion of the Classification Theorem.) The main question left open, whether Casson handles might actually be diffeomorphic to open 2-handles, was answered negatively by Donaldson's contemporaneous work (yielding another Fields Medal): one cannot usually even find a smoothly embedded disk in a Casson handle, bounded by the attaching circle in $\partial_{-} \mathrm{CH}$; otherwise the high-dimensional machinery would run smoothly, contradicting many results from gauge theory. Fortunately, Donaldson's work did not precede Freedman's; otherwise topological 4-manifold theory might never have been developed.

The Casson handle embedding theorem that we will need is taken from Quinn's paper [20, Proposition 2.2.4]. A smooth 4-manifold $V$ with a circle $C$ in its boundary will be called a possibly exotic 2-handle with attaching circle $C$ if there is a homeomorphism $h:\left(D^{2} \times \mathbb{R}^{2}, S^{1} \times\{0\}\right) \rightarrow(V, C)$, and $h\left(D^{2} \times\{0\}\right) \subset V$ will be called a topological core. (This core is unique up to topological ambient isotopy.) Since Casson handles are examples, we cannot in general expect to have a smoothly embedded 2-disk in $V$ bounded by $C$, and so $h$ cannot usually be smoothed near $D^{2} \times\{0\}$. However, we do obtain the following:

Theorem $5.2[\mathbf{2 0}]$. A possibly exotic 2-handle $(V, C)$ always contains an unknotted Casson handle $\mathrm{CH}$ attached along $C$. 
Here, unknotted means that the topological core of CH given by Freedman's theorem is topologically ambiently isotopic in $V$ (rel $C$ ) to the topological core $h\left(D^{2} \times\{0\}\right)$ of $V$. Quinn had to settle for a "weakly unknotted" Casson handle; we prove the stronger statement using more modern technology. This theorem was originally assumed to be of limited use, since it only gives a Casson handle where we already have a topological 2-handle. Basically, it only replaces an arbitrary exotic 2-handle by a slightly better understood exotic 2-handle. However, we will find this upgrade crucial for creating Stein thickenings, and Quinn's proof will be an important part of our construction for Theorem 2.4.

Proof. While the given homeomorphism $h: D^{2} \times \mathbb{R}^{2} \rightarrow V$ cannot be smoothed near $D^{2} \times\{0\}$, Quinn uses Freedman theory to investigate where $h$ can be smoothed. His Handle Straightening theorem [20, 2.2.2] is based on showing that a homeomorphism can be smoothed (isotoped to a diffeomorphism) outside of a small neighborhood of a smooth 2-complex with special properties. Thus a homeomorphism of an open 0- or 1-handle that is a diffeomorphism on the boundary can be smoothed near its core, simply by smoothly isotoping the core away from the 2-complex via transversality, whereas this fails for 2-handles. In the latter case, however, his theorem shows that we can avoid the problematic 2-complex by smoothly homotoping $D^{2} \times\{0\}($ rel $\partial)$ to a suitable generically immersed disk $D \subset D^{2} \times \mathbb{R}^{2}$. Then we can assume $h$ is a diffeomorphism near $D$, so $h(D)$ is a smoothly immersed disk in $V$ with $\partial D=C$.

The advantage of using $h(D)$, rather than any generic smoothing of the map $h \mid D^{2} \times\{0\}$, is that the kinky handle $\kappa$ in $D^{2} \times \mathbb{R}^{2}$ obtained by thickening $D$ can be turned into a 2 -stage tower with no kinks at the second stage - and furthermore, this tower in $D^{2} \times \mathbb{R}^{2}$ is smoothly ambiently isotopic to a closed tubular neighborhood of $D^{2} \times\{0\}$ (so we preserve unknottedness). To see this, note that Quinn's homotopy consists of finger moves to push $D^{2} \times\{0\}$ off the bad set. These moves consist of "pushing the disk with one finger," along some arc $\gamma$ and back through itself as in Figure 4, creating an extra pair of intersections. Finger moves, which can be thought of as inverses of Whitney moves, have been used extensively for cleaning up immersed

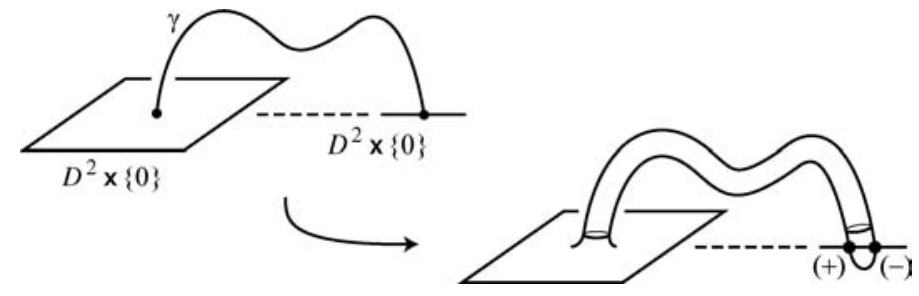

Figure 4. Finger move. 
surfaces since Casson's work. A finger move is determined by the arc $\gamma$ with endpoints on the surface, together with a normal vector field to $\gamma$, whose orthogonal complement in $\nu \gamma$ carries the circle bundle comprising the tube around our finger. (Automorphisms of the circle bundle do not affect the image set $D$.) Since any two nonzero sections of the 3-plane bundle $\nu \gamma$ are homotopic rel boundary if they agree on the boundary, $D$ is determined by the $\operatorname{arcs} \gamma_{i}$ specifying the finger moves. In fact, this collection of arcs is (in our application) smoothly isotopic to a standard model. This is because 1-manifolds cannot knot or link in a 4-manifold (homotopy implies isotopy) and the corresponding homotopy problem is trivial: once we isotope $\gamma_{i}$ so that it agrees near its endpoints with the corresponding model $\operatorname{arc} \gamma_{i}^{\prime}$, the two arcs determine an element of $\pi_{1}\left(D^{2} \times \mathbb{R}^{2}-D^{2} \times\{0\}\right) \cong \mathbb{Z}$. However, we can change this element by a generator, by turning $\gamma_{i}$ once around the normal fiber to $D^{2} \times\{0\}$ at one endpoint of $\gamma_{i}$. Thus we can assume $\gamma_{i}$ and $\gamma_{i}^{\prime}$ are homotopic, hence isotopic. Now that we have reduced to considering a standard model of a finger move, it is straightforward to exhibit the required pair of disks.

We can now complete Quinn's construction. Applying the homeomorphism $h$ to our 2-stage tower in $D^{2} \times \mathbb{R}^{2}$, we obtain towers $h(\kappa)=T_{1} \subset$ $T_{2}^{*} \subset V$. While $T_{1}$ is a smooth 1 -stage tower (kinky handle), our new disks in $D^{2} \times \mathbb{R}^{2}$ hit the nonsmooth part of $h$, so the second stage of $T_{2}^{*}$ consists of topologically embedded 2-handles $h_{i}$. Stripping off the boundary of each $h_{i}$ (except for int $\partial_{-} h_{i}$, which is smooth), we obtain possibly exotic 2-handles (in the smooth structure inherited from $V$ ), so we can repeat the previous construction in each $h_{i}$. Inductively assume we have towers $T_{n} \subset T_{n+1}^{*} \subset V$, where the $n$-stage tower $T_{n}$ is smooth, and $T_{n+1}^{*}$ is obtained from it by adding topologically embedded 2-handles. Then $T_{n+1}^{*}$ is homeomorphic to a 2-handle (since there are no top-stage kinks); also assume $T_{n+1}^{*}$ is topologically ambiently isotopic to a closed tubular neighborhood of the topological core disk of $V$. Applying the previous construction to the possibly exotic 2-handles of the top stage, we recover the induction hypotheses with $T_{n} \subset T_{n+1} \subset T_{n+2}^{*} \subset T_{n+1}^{*}$. Taking the infinite union of $T_{1} \subset T_{2} \subset T_{3} \subset \cdots$ and removing the boundary except for int $\partial_{-} h(\kappa)$, we obtain a smoothly embedded Casson handle $\mathrm{CH} \subset V$ with attaching circle $C$.

To prove $\mathrm{CH} \subset V$ is unknotted, let $\Delta$ be a topological core disk of $\mathrm{CH}$. Working in the topological category, we add boundary to $V$ to obtain $\Delta \subset$ $D^{2} \times D^{2}=B^{4}$, with $V$ identified with $D^{2} \times$ int $D^{2}$. We know that $(\Delta, \partial \Delta) \subset$ $\left(B^{4}, \partial B^{4}\right)$ is flat, i.e., it is the core of an embedded 2-handle $\left(D^{2} \times D^{2}, S^{1} \times\right.$ $\left.D^{2}\right) \hookrightarrow\left(B^{4}, \partial B^{4}\right)$ (obtained from the product structure of $\mathrm{CH} \approx D^{2} \times$ $\mathbb{R}^{2}$ ), whose interior we denote by $W \approx D^{2} \times$ int $D^{2}$. We wish to show that $\pi_{1}\left(B^{4}-\Delta\right) \cong \mathbb{Z}$, for then Freedman's work immediately shows that $\Delta$ is unknotted in $B^{4}$ and hence in $V$ : in fact, $B^{4}-W$ is a topological 4manifold with boundary $S^{2} \times S^{1}$ (since $\partial \Delta=C \subset \partial B^{4}$ is unknotted), so the 
$s$-Cobordism Theorem with $\pi_{1} \cong \mathbb{Z}[\mathbf{1 1}]$ identifies $B^{4}-W$ with $B^{3} \times S^{1}$, and this uniquely glues to $W$. Since $H_{1}\left(B^{4}-\Delta\right) \cong \mathbb{Z}$ (e.g., by MayerVietoris), it suffices to show that any nullhomologous loop $\gamma$ in $B^{4}-\Delta$ is nullhomotopic. Compactness of $\Delta \subset \mathrm{CH}$ guarantees that $\Delta$ lies in some subtower $T_{n}$ of $\mathrm{CH}$. By construction, $T_{n}$ lies in $T_{n+1}^{*}$, which is a deformation retract of $B^{4}$ (since it is isotopic to a closed tubular neighborhood of the core of $V$ ). Thus, we can assume $\gamma$ lies in $T_{n+1}^{*}-\Delta$. Now we push $\gamma$ off of the top-stage 2-handles of $T_{n+1}^{*}$, so that $\gamma$ lies in int $T_{n}-\Delta \subset \mathrm{CH}-\Delta$. But inclusion $\mathrm{CH}-\Delta \hookrightarrow B^{4}-\Delta$ induces an $H_{1}$-isomorphism, since both groups are $\mathbb{Z}$ generated by a meridian of $\Delta$. Since $\gamma$ is nullhomologous in $B^{4}-\Delta$, it is then nullhomologous in $\mathrm{CH}-\Delta$, and hence nullhomotopic (since $\mathrm{CH}-\Delta \approx D^{2} \times\left(\mathbb{R}^{2}-\{0\}\right)$ has abelian $\left.\pi_{1}\right)$.

\section{Proof of main Theorem 2.4}

We prove Theorem 2.4 by combining the methods of the two previous sections. Recall that we are given a topologically embedded 2-complex $K$ in a complex surface $X$, and $K$ is tame, i.e., it can be thickened to a handlebody. We wish to move $K$ by an $\varepsilon$-small topological ambient isotopy, so that it has an $\varepsilon$-small Stein thickening. To make the construction $\varepsilon$-small, we simply subdivide $K$ as necessary and work within the resulting small handles of a thickening. The 1-skeleton of $K$ is still easy to deal with: by Quinn's Handle Straightening Theorem $[\mathbf{2 0}, 2.2 .2]$, we can assume the 0 -handles are embedded by homeomorphisms that are smooth near the vertices of $K$, then make them Stein as in Section 4 (by a topological ambient isotopy fixing $K$ outside the 0-handles). Similarly, we can assume (after an $\varepsilon$-small topological isotopy) that the 1-handles are smooth near the cores, then modify them as in Section 4. We have now topologically isotoped $K$ so that the 1-skeleton is smooth and has a small Stein thickening $U_{1}$, with $\partial U_{1}$ a level set of a plurisubharmonic function $\varphi$, and $U_{1}$ extends to a topological thickening of all of $K$. (If $K$ is infinite, we again work with only finitely many handles at a time, starting on 2-handles before finishing the 1-handles, to maintain properness of $\varphi$.)

The main challenge is the 2-cells. Each 2-cell determines a topological embedding $f: D^{2} \rightarrow X$ that is smooth near $f^{-1}\left(\partial U_{1}\right)=\partial D^{2}$, and the image is the core of a possibly exotic 2-handle. The proof of Theorem 5.2 transforms $f$ into a smooth immersion $f^{\prime}$ such that the induced kinky handle has second stage topologically embedded 2-handles for reconstructing (up to topological isotopy) a thickening of $f\left(D^{2}\right)$. After we clean up near $\partial f^{\prime}\left(D^{2}\right)$ as in Section 4, the obstructions of Theorem 3.1 are defined for $f^{\prime}$. As before, we can assume the Chern class obstruction vanishes, so the 


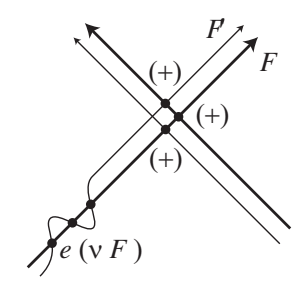

Figure 5. Homological self-intersection number.

remaining obstruction $e(\nu)+1$ is even. Recall that the fundamental difficulty of the method of Section 4 was the failure of this Euler class obstruction to vanish in general. However, we are no longer constrained to work with embedded surfaces. For immersed surfaces, we can change $e(\nu)$ by \pm 2 , simply by adding a kink with sign $\mp 1$. (Note that for a closed, oriented, generically immersed surface $F$, its homological self-intersection number is $F \cdot F=e(\nu F)+2 \operatorname{Self} F$, where Self $F$ is the signed number of double points of $F$. This follows by pushing off a copy $F^{\prime}$ of $F$ and counting intersections with $F$ as in Figure 5 to obtain $F^{\prime} \cdot F=F \cdot F$. Since $F \cdot F$ is a homological invariant, adding a $\mp$ kink to $F$ must change $e(\nu F)$ by \pm 2 .) Thus, we can make $e(\nu)+1$ vanish by adding kinks to $f^{\prime}\left(D^{2}\right)$. Each kink can be added in a standard chart $\left(\mathbb{R}^{4}, \mathbb{R}^{2}\right)$ using a standard model that has a smoothly embedded second-stage 2-handle. Thus, the resulting immersion $f^{\prime \prime}$ still has the second stage required for Quinn's construction. We can easily adjust $f^{\prime \prime}$ near each double point so that the two sheets correspond to $\mathbb{R}^{2}$ and $i \mathbb{R}^{2}$ in a holomorphic local chart, then apply Theorem 3.1 away from the double points to make $f^{\prime \prime}$ totally real. A $C^{1}$-small perturbation, fixing $f^{\prime \prime}$ and $d f^{\prime \prime}$ on $\partial D^{2}$ and at the double points, makes $f^{\prime \prime}$ real-analytic and totally real, with multiplication by $i$ interchanging the tangent directions at each double point. Theorem 1.3 .5 of [5] says that totally real immersions, with $i$-invariant double points as above, have Stein regular neighborhoods, and its proof allows us to extend $\varphi$ over a kinky handle $\kappa$ with core $f^{\prime \prime}(D)$, so that $\partial \kappa-\partial_{-} \kappa$ lies in a level set.

To complete the construction, we apply induction as for Theorem 5.2, while at each stage adding a thin collar and maintaining the Stein condition as above. We proceed with the cells of $K$ and tower stages ordered by $\mathbb{Z}^{+}$so that each stage of the construction is a finite handlebody, but each handle and kinky handle are eventually included. The union $U$ of these handlebodies is itself an open topological handlebody, whose 1-skeleton int $U_{1}$ is a thickening of the 1-skeleton of $K$ (which we have already adjusted by a topological isotopy). The 2-handles of $U$ are Casson handles, one for each 2-cell of $K$. By the unknottedness assertion of Theorem 5.2, each 2-cell is topologically ambiently isotopic rel $\partial$ to the core of the corresponding 
Casson handle, so after a topological ambient isotopy of $K, U$ becomes a topological thickening of $K$. (For infinite $K$, we can ensure that the ambient isotopy extends from the original thickening $V$ to all of $X$, by taking the preassigned $\varepsilon$ to be a continuous function with $\varepsilon \mid V>0$ but $\varepsilon \mid X-V=0$.) Since $U$ was constructed with a proper plurisubharmonic function, it is Stein in the complex structure inherited from $X$, so it is the required thickening completing the proof of Theorem 2.4.

\section{Examples 6.1.}

(a) For a smooth 2-knot $K$ in $\mathbb{C}^{2}$, we have $c_{1}\left(\mathbb{C}^{2}\right)=0, e(\nu K)=K \cdot K=0$, and $\chi(K)=2$. Thus, we can make the sphere $K$ totally real by adding a single positive kink. We can do this with a standard model kink so that the second stage disk can also be made real with a single positive model kink. By induction, one obtains a Stein thickening $U$ of $K$, after a $C^{0}$-small topological ambient isotopy that is smooth except near the kink, where $U$ is obtained by adding the simplest Casson handle (with one positive kink at each stage) to a 4-ball along an unknot (with framing 0). This is a universal diffeomorphism type for Stein thickenings of smooth 2-knots, in the sense of Example 2.5, since we have just described $U$ up to diffeomorphism by data independent of $K$. (The diffeomorphism type of a Casson handle is determined by the number and signs of the kinks of the kinky handles. This data can be expressed by a based tree with a sign attached to each edge.) The minimal genus of the generator of $H_{2}(U)$ is 1 , since $U$ cannot contain a smooth essential sphere, but the immersed sphere can be smoothed to an embedded torus.

(b) More generally, for any smooth embedding $F \hookrightarrow X$ of a closed, oriented, connected surface into a (possibly noncompact) complex surface with $\left\langle c_{1}(X), F\right\rangle=0$, we must add $m=\frac{1}{2} \max (0, F \cdot F+\chi(F))$ positive kinks to the 2-cell to make it totally real. (Recall that instead of negative kinks, we can suitably adjust the Legendrian boundary when $F \cdot F+\chi(F)<0$.) We obtain a Stein thickening where the Casson handle attached to the 1-skeleton has only positive kinks, with $m$ at the first stage and one on each higher stage kinky handle. (For $m=0$, this is just a smooth 2-handle, and the resulting Stein tubular neighborhoods can also be obtained by another method $[\mathbf{8}, \mathbf{9}]$.) This diffeomorphism type is then universal for all such embeddings of $F$ (with $F \cdot F$ fixed). When $\left\langle c_{1}(X), F\right\rangle \neq 0$, we must absorb this obstruction with extra spirals in the Legendrian boundary, so the above holds with $m=\frac{1}{2} \max \left(0, F \cdot F+\chi(F)+\left|\left\langle c_{1}(X), F\right\rangle\right|\right)$. We obtain a universal diffeomorphism type $U_{g, k, c}$ of Stein thickenings for smooth embeddings with $g(F)=g, F \cdot F=k$, and $\left|\left\langle c_{1}(X), F\right\rangle\right|=c$. Note that we can 
realize any $g, c \geq 0$ and $k \equiv c \bmod 2$ by such embeddings, by immersing $F$ with degree 1 in a holomorphic line bundle realizing $g$ and $c$, then taking $X$ to be a pulled back tubular neighborhood of $F$. The minimal genus of $U_{g, k, c}$ is $g+m$ (except conceivably when $g=0, m=1$, and $k \leq-2$ ), by the genus bound of [17] (see also [14, Theorem 11.4.7]). To realize universal diffeomorphism types of all larger minimal genera (for fixed $g, k, c)$, simply use $U_{g, k, c+2 n}$ for all $n>0$. These can be substituted for $U_{g, k, c}$ by adding extra positive kinks at the first stage of the construction and compensating by suitable spirals in the attaching circle. For example, we obtain universal diffeomorphism types of any nonzero genus in Example 2.5(a).

(c) Consider topological embeddings $F \hookrightarrow X$ that can be thickened (to a homeomorphism of the 2-plane bundle over $F$ with Euler number $F \cdot F$ ). Now our construction requires Quinn's Handle Straightening Theorem, so it is much harder to extract information about the complexity of the resulting Casson handle. For any fixed $g, k, c$ as above, we have topological embeddings $F \hookrightarrow X_{n}=U_{g, k, c+2 n}$ for which the minimal genus realizing $[F] \in H_{2}\left(X_{n}\right)$ is arbitrarily large. Thus, there cannot be a universal diffeomorphism type for this problem. However, we will see that there is a fixed diffeomorphism type of $U$ for any finite collection of such embeddings with fixed $g, k$.

\section{More tricks}

The machinery of topological 4-manifold theory developed over a period of more than a decade, and many specialized techniques arose in the process. One can obtain much more information about Stein thickenings by reviewing these techniques and inserting the word "Stein" in various places. We sketch some ideas here and will return to the matter in $[\mathbf{1 3}]$.

We first observe that any two Casson handles have a common refinement: Simply compare the Casson handles, from the first stage up, and add kinks of suitable sign to each whenever needed to produce a new Casson handle smoothly embedded in both. (Equivalently, one constructs a signed tree containing both of the original signed trees.) Given a finite collection of topological embeddings $K \hookrightarrow X_{i}$, with homeomorphic (rel $K$ ) thickenings, we immediately see how to construct Stein thickenings for them that are diffeomorphic to each other: for each 2-cell of $K$, construct the corresponding Casson handles in each $X_{i}$ simultaneously, adding extra kinks where necessary so that the resulting Casson handles all have the same signed tree, hence are diffeomorphic. (A sufficient excess of positive kinks everywhere guarantees that the constructed thickenings can be made Stein.) We can also enforce lower bounds on minimal genera by this method, refining the

Stein thickening so that it smoothly embeds somewhere else where a genus 
bound for the homology class is known. For example, given $K \subset X$ as usual and $\alpha \neq 0 \in H_{2}(K)$ (if this exists), Theorem 2.3 implies the thickening of $K$ is homeomorphic to a Stein surface $V$ for which $\left\langle c_{1}(V), \alpha\right\rangle$ exceeds any preassigned value, and hence the minimal genus of $\alpha$ in $V$ exceeds a preassigned value ([14] Exercise 11.4.11(d) and solution). A sufficiently refined Stein thickening $U$ for $K$ smoothly embeds in $V$ (not preserving $c_{1}$ ), so the minimal genus of $\alpha$ in $U$ also exceeds the preassigned value. This explains the genus bound of Example 2.5(b).

While smoothly embedded complexes provide a rich source of examples for Theorem 2.4, we would also like to locate nonsmooth examples. One good method is Casson's original theorem for embedding Casson handles [2]. This shows, for example, that in a simply connected 4-manifold $X$, any class of square \pm 1 in $H_{2}(X)$ whose orthogonal complement is odd can be represented by a topological embedding of $\pm \mathbb{C P}^{2}-\{p\}$ (hence, a tame topological $S^{2}$ ), and any hyperbolic pair $\left[\begin{array}{ll}0 & 1 \\ 1 & 0\end{array}\right]$ in the intersection form can be represented by an embedding of $S^{2} \times S^{2}-\{p\}$ (so a tame $S^{2} \vee S^{2}$ ). (These can also be deduced from Freedman's Classification Theorem if $X$ is closed.) If $X$ is a closed, minimal complex surface with $b^{+}>1$, gauge theory shows that these embeddings can never be made smooth, but Theorem 2.4 makes the images Stein.

Next we consider exotic $\mathbb{R}^{4}$ 's. The basic constructions of these are in Casson's original paper, although he could not draw the final conclusions with neither Freedman nor Donaldson theory yet available. The general class of exotic $\mathbb{R}^{4}$ 's containing those of Theorem 2.6 appeared in [4], while the simplest case of these was shown to be exotic in [1] (see also [14]) and to be Stein in [12]. These simplest examples are constructed with one 0-handle, two 1-handles, one 2-handle, and a Casson handle. The range of diffeomorphism types comes from varying the complexity of the Casson handle. To embed such an example as a Stein open subset of $\mathbb{C}^{2}$, we must first embed the honest handles. While it is easy to embed the 1-skeleton as a Stein surface, it takes work to arrange the 2-cell so that the obstructions of Theorem 3.1 can both be explicitly seen to vanish. (Details will appear in [13].) Once this is achieved, the Casson handle can be located by Casson's Embedding Theorem, and our previous techniques yield the Stein surface.

One of the main ingredients of the proof of Freedman's Theorem 5.1 is his reimbedding theorems. These elucidate the structure of a Casson handle $\mathrm{CH}$ by finding other Casson handles with compact closure inside. Ultimately, Freedman obtains an uncountable family of Casson handles indexed by a standard Cantor set $C \subset I=[0,1]$, nested with compact closure inside each other with the ordering inherited from $C$. Instead of a Casson handle at $0 \in C$, Freedman shows that the intersection of the remaining Casson handles can be taken to be a topological core disk $\Delta$ of $\mathrm{CH}$, and $\Delta$ can be assumed smooth except at a single interior point. The main principle of our 
Section 6 is that in a complex surface, Casson handles can be assumed to preserve Stein structures, as long as we are allowed to add enough positive kinks. We conclude that any Stein surface $U$ made with finitely many handles and Casson handles contains a family of Stein surfaces homeomorphic to $U$, nested with compact closure, with the order type of $C-\{0\}$. (For example, any $U$ arising from a finite handlebody in Theorem 2.3 or a compact $K$ in Theorem 2.4 has this structure.) The intersection of these nested Stein surfaces will be a tame 2-complex, smoothly embedded except at a single point in the interior of each 2-cell, and the 2-cells will be totally real where they are smooth. In the case of the above exotic $\mathbb{R}^{4},[\mathbf{4}]$ shows that each diffeomorphism type appears only countably often within the nested family, so there are uncountably many diffeomorphism types of Stein exotic $\mathbb{R}^{4}$ 's realized this way in $\mathbb{C}^{2}$, with the cardinality of the continuum in ZFC set theory (proving Theorem 2.6). The argument of [4] is too specialized to apply to all Stein surfaces, but in some cases (e.g., any open subset of $\mathbb{C}^{2}$ ) we can distinguish uncountably many diffeomorphism types after we connect by a 1-handle to the family of Theorem 2.6 (proving Theorem 2.7). Other methods sometimes distinguish all diffeomorphism types in a nested family as above, e.g., for Stein surfaces homeomorphic to $\pm \mathbb{C P}^{2}-\{p\}$ or $S^{2} \times S^{2}-\{p\}$. Our nesting procedure can be combined with genus restrictions, so that the minimal genus of a class $\alpha \neq 0 \in H_{2}(U)$ increases without bound as the depth in the nesting increases. Alternatively, we can arrange the minimal genus of $\alpha$ to be constant, by locating a surface $F$ realizing $\alpha$ with minimal genus in $U$, observing that for some $n, F$ lies in the union of the 1 -skeleton and $n$-stage towers of $U$, then applying reimbedding only to the Casson handles attached on top of the $n$-stage towers. Note that neighborhood systems of homotopy-equivalent Stein surfaces are of interest to complex analysts (e.g., $[\mathbf{8}, \mathbf{9}]$ for neighborhood systems of smoothly embedded surfaces), but such neighborhoods are more typically diffeomorphic to each other.

The family of exotic $\mathbb{R}^{4}$ 's in $[4]$ is actually parametrized by $I$, with $C \subset I$ corresponding to exotic $\mathbb{R}^{4}$ 's made with reimbedding technology. To interpolate to a connected family in this way, one must know that the point-set boundaries of the nested $\mathbb{R}^{4}$ 's are homeomorphic to $S^{3}$ (concentric round spheres in the topological $\mathbb{R}^{4}$ structure). Unfortunately, embedded Casson handles typically have point-set boundaries that are not manifolds. (We can at least arrange that the complements of their closures have the same homotopy groups as the complements of their cores, by sharpening the method of proof of Theorem 5.2, but otherwise the boundaries are ugly.) To fix this, one replaces Casson handles by the infinite towers of capped gropes in [11]. This means that between successive stages of immersed disks, one inserts many stages of embedded surfaces. This cleans up the boundaries, so that the topological $D^{2} \times$ int $D^{2}$ can be parametrized with the radial coordinates 
corresponding to $C \subset I$ realized by reimbedded towers. We can apply this technology in the Stein setting, since increasing genus has the same effect on $e(\nu F)+\chi(F)$ as adding positive kinks, but there are more technical details. (For example, the towers of [11] are constructed, for convenience, with the same number of positive and negative kinks in each kinky handle, whereas we need an excess of positive kinks.) The final result is that the families of Stein surfaces parametrized by a Cantor set lie in continuous 1-parameter families of homeomorphic open sets (not necessarily Stein for parameter values in $I-C)$.

Our final observation on Stein surfaces is that Theorem 2.3 can be deduced from Main Theorem 2.4. (Of course, their proofs rely on similar ideas in any case.) If $X$ is the interior of a handlebody whose indices are all $\leq 2$, and $J$ is an almost-complex structure on $X$, there is a map $f$ from $X$ to the rational surface $\mathbb{C P}^{2} \# \overline{\mathbb{C P}}^{2}$, covered by a complex bundle map of the tangent bundles. (Send the 1-skeleton to a point and wrap each 2-cell around the generators as necessary to preserve $c_{1}$.) By immersion theory, we can homotope $f$ to an immersion, then pull back the complex structure, to create a complex structure on $X$ homotopic to $J$. If $K \subset X$ is the 2-complex obtained from the cores of the handles, applying Theorem 2.4 to $K$ gives the required Stein surface.

Since Stein surfaces are in some sense equivalent to Weinstein 4-manifolds in the symplectic category, it is natural to ask whether there is an analog of Theorem 2.4 for identifying open subsets of symplectic 4-manifolds that are Weinstein. The main difference is that cores of 2-handles are Lagrangian in the Weinstein setting - a closed condition that is strictly stronger than the totally real condition for Stein 2-handles. Fortunately, there is an $h$-principle for immersed Lagrangian submanifolds, so we can still obtain immersed disks by killing the invariants of Theorem 3.1. It is not presently clear to the author, however, whether these immersions can be created with sufficient control to allow Quinn's tower construction to proceed. The author hopes to return to this question soon.

\section{References}

[1] Ž. Bižaca and R. Gompf, Elliptic surfaces and some simple exotic $\mathbb{R}^{4}$ 's, J. Diff. Geom. 43 (1996), 458-504.

[2] A. Casson, Three lectures on new infinite constructions in 4-dimensional manifolds (notes prepared by L. Guillou), A la Recherche de la Topologie Perdue, Progress in Mathematics, 62, Birkhäuser, 1986, 201-244.

[3] S. Chern and E. Spanier, A theorem on orientable surfaces in 4-dimensional space, Comm. Math. Helv. 25 (1951), 205-209.

[4] S. DeMichelis and M. Freedman, Uncountably many exotic $\mathbb{R}^{4}$ 's in standard 4-space, J. Diff. Geom. 35 (1992), 219-254. 
[5] Y. Eliashberg, Topological characterization of Stein manifolds of dimension $>2$, Int. J. Math. 1 (1990), 29-46.

[6] Y. Eliashberg and M. Gromov, Convex symplectic manifolds, Proc. Symp. Pure Math., 52, (Part 2), 135-162, Am. Math. Soc., Providence, RI, 1991.

[7] Y. Eliashberg and V. Harlamov, Some remarks on the number of complex points of a real surface in a complex one, Proc. LITC-82, 143-148.

[8] F. Forstnerič, Complex tangents of real surfaces in complex surfaces, Duke Math. J. 67 (1992), 353-376.

[9] F. Forstnerič, Stein domains in complex surfaces, J. Geom. Anal. 13 (2003), 77-94.

[10] M. Freedman, The topology of four-dimensional manifolds, J. Diff. Geom. 17 (1982), 357-453.

[11] M. Freedman and F. Quinn, Topology of 4-Manifolds, Princeton Math. Ser., 39, Princeton Univ. Press, Princeton, NJ, 1990.

[12] R. Gompf, Handlebody construction of Stein surfaces, Ann. Math. 148 (1998), 619-693.

[13] R. Gompf, Creating Stein surfaces by topological isotopy, (In preparation).

[14] R. Gompf and A. Stipscz, 4-manifolds and Kirby calculus, Grad. Studies Math., 20, Am. Math. Soc., Providence, 1999.

[15] H. Grauert, On Levi's problem, Ann. Math. 68 (1958), 460-472.

[16] P. Kronheimer and T. Mrowka, Monopoles and contact structures, Invent. Math. 130 (1997), 209-255.

[17] P. Lisca and G. Matić, Tight contact structures and Seiberg-Witten invariants, Invent. Math. 129 (1997), 509-525.

[18] J. Milnor, Morse Theory, Ann. Math. Stud. 51, Princeton Univ. Press, 1963.

[19] S. Nemirovski, Complex analysis and differential topology on complex surfaces, Russian Math. Surveys 54:(4), (1999), 729-752.

[20] F. Quinn, Ends of maps. III: dimensions 4 and 5, J. Diff. Geom. 17 (1982), 503-521.

[21] L. Taylor, An invariant of smooth 4-manifolds, Geom. Topol. 1 (1997), 71-89.

[22] A. Weinstein, Contact surgery and symplectic handlebodies, Hokkaido Math. J. 20 (1991), 241-251.

Department of Mathematics

The University of Texas at Austin

1 University Station C1200

Austin, TX 78712-0257

E-mail address: gompf@math.utexas.edu

Received 01/29/2005, revised 06/01/2005. Partially supported by NSF grant DMS0102922 . 
